\title{
A SPOT WELDING RELIABILITY PROBLEM
}

\author{
M. RUMSEWICZ ${ }^{1}$ AND P. TAYLOR
}

(Received 30 October 1986; revised 16 December 1986)

\begin{abstract}
When multiple operators are connected to a single power source which is not large enough to simultaneously supply all users, interference can take place. This paper considers two models of a spot welding station which differ in the method of resolving interference between users. In the first model the system is allowed to become overloaded and the consequent deterioration in weld quality is accepted. In the second model any request which will overload the system is rejected. Expressions are found for (a) the proportion of poor quality welds in the first model and (b) the probability of operators being rejected in the second model. Numerical results are given which indicate how small the power supply can be made for a typical welding shop while keeping interference at a minimal level.
\end{abstract}

\section{Introduction}

Many production lines have a station where spot welding takes place. At such stations it is frequently the case that multiple operators draw on the same power supply. Given the high voltage drops that occur during spot welding and the deterioration in weld quality that can occur when a large number of operators spot weld simultaneously, it is of interest to investigate the probability of poor welds being produced.

The time taken to perform a single spot weld is small compared to the period between consecutive welds by one operator and hence it might be expected that the probability of many welders simultaneously drawing on the power supply is small. As a consequence, it may be possible to install a power supply that can only support some of the welders at any given time without significantly affecting the performance of the welding shop.

\footnotetext{
'Department of Applied Mathematics, University of Adelaide, G. P. O. Box 498, Adelaide, S. A. 5001, Australia.

(C) Copyright Australian Mathematical Society 1988, Serial-fee code 0334-2700/88
} 
If a smaller power supply is installed then there are two possible methods for dealing with any interference which may take place. Firstly, any number of simultaneous welds may be allowed to take place, thus accepting the possibility of bad welds. Defective welds pass undetected by the operators and no attempt is made to rectify them. Secondly, access to the power supply may be restricted so overloading does not occur. The second alternative will involve operators having to make repeated attcmpts to weld. Providing this does not occur often, this may be preferable from a reliability point of view.

This paper looks at queueing models for the two alternatives described above. Section 2 considers the case where there is no restriction on the power supply, so that it is possible for all operators to weld simultaneously. Expressions are derived for the probabilities of the power supply simultaneously supplying multiple operators. In contrast, Section 3 looks at a modification of the system where the number of simultaneous welds is restricted. In Section 4, the results of Sections 2 and 3 are used to discuss the efficiency of the two systems.

\section{The unrestricted system}

Consider a spot welding station with $N$ operators. To complete a single spot weld takes a fixed time of $\mu^{-1}$ seconds. The time between successive welds of a single operator has distribution function $G(\cdot)$ with mean $\lambda^{-1}$. The power supply is such that all operators may access it at the same time. However, if more than $K$ operators actually use it simultaneously, the quality of all welds currently in progress deteriorates.

The system can be considered as a closed two-node queueing network with $N$ customers (see Figure 1). Those customers in node 1 are welding while those in

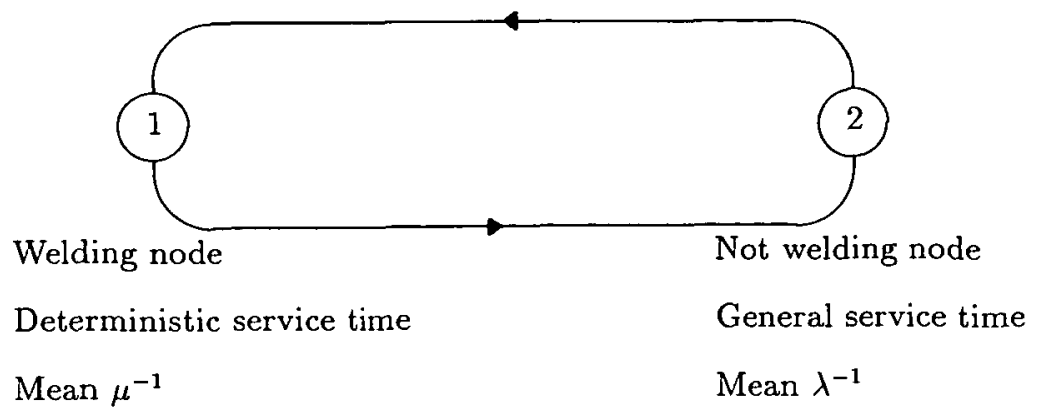

$N$ welders

Figure 1. Two node model of a spot welding shop. 
node 2 are not welding. Both nodes have $N$ servers, with the service time at node 1 being fixed and of duration $\mu^{-1}$ seconds and the service time at node 2 having distribution $G(\cdot)$ with mean $\lambda^{-1}$ seconds.

This is an example of an Engset system having $N$ servers and a population of size $N$, with service and interarrival time distributions as given above. This system has been studied by König [2] who showed that the steady-state queue-length distribution only depends on the service and interarrival time distributions through their means, i.e. the steady state distribution is insensitive to the form of the service and interarrival time distributions.

Hence, the steady-state distribution may be found by considering the system with negative exponential distributions. This gives

$$
p_{\imath}=\frac{\left(\frac{\lambda}{\mu}\right)^{i}\left(\begin{array}{c}
N \\
i
\end{array}\right)}{\left(1+\frac{\lambda}{\mu}\right)^{N}}
$$

The expected number of bad welds at any given instant is

$$
N_{b}=\sum_{i=K+1}^{N} i p_{i}
$$

whereas the expected number of welds in progress at any given instant is

$$
N_{w}=\sum_{i=1}^{N} i p_{\iota}=\frac{N \lambda}{\lambda+\mu} .
$$

Hence the proportion of bad welds is $N_{b} / N_{w}$.

\section{The restricted system}

Now consider the system described in Section 2, but with the restriction that the electricity supply is limited to perform only $K \leqslant N$ spot welds simultaneously, $K$ being the maximum number of welds that can be performed at the same time without a drop in quality. If an operator tries to spot weld while $K$ other welds are already in progress, the attempt is rejected and the operator must try again. The process is repeated until there is a successful attempt.

Restricting the power supply as described above reduces the incidence of low-quality welds at the expense of occasionally denying access to the power supply for some of the operators. However, if the probability of denying access is 
small, then the efficiency of the welding shop is little affected but its reliability is increased.

In modelling the restricted system the following assumptions are made:

(a) the service time (duration of a spot weld) is negative exponentially distributed with mean $\mu^{-1}$ seconds,

(b) for each operator, the period between completing a weld and the next attempt is negative exponentiaily distributed with mean $\lambda^{-i}$ seconds,

(c) for each operator, the period between an unsuccessful attempt to weld and the next attempt is negative exponentially distributed with mean $\gamma^{-1}$ seconds.

Note that in practice $\gamma^{-1}$ is likely to be small compared to $\lambda^{-1}$, since operators denied access are likely to reattempt quickly.

Even though service times are known to be deterministic and it is unlikely that the distribution of time until a reattempt is negative exponential, the distributions used here have been assumed to be negative exponential. This has been done to aid solution of the problem. When the probability of an operator being rejected is small, this is a good assumption since the system closely approximates the model analysed in Section 2, the solution of which is insensitive to the form of the distributions.

Supplement the state space of Section 2 by including a component to record the number of operators that have made an unsuccessful attempt to weld. The state of the system when $n$ welds are being performed and $j$ operators have made an unsuccessful attempt and are trying again is $(n, j)$. Thus, when the state is $(n, j)$, reattempts are made at rate $j \gamma$, and if $n<K$ the reattempt is successful and the state becomes $(n+1, j-1)$. If $n=K$ and another operator (i.e. one that is not reattempting) tries to weld, then the state moves to $(K, j+1)$. This happens at rate $(N-K-j) \lambda$. Denote by $p_{n j}$ the probability of being in state $(n, j)$.

Figure 2 is the state transition diagram, marked with the appropriate transition rates. Let the set of states $\{(n, j) ; 0 \leqslant j \leqslant N-K\}$ be denoted by the level $n$. This allows the system to be considered as a quasi birth and death process (see Neuts [3]), whereby transitions with positive rate cause the level to change by at most one up or down. It may be noted that $j$, the number of delayed welders, may be considered as the state of the environment (see Neuts [3], Gaver et al. [1]).

It is clear that the process has a block tri-diagonal $Q$-matrix,

$$
Q=\left[\begin{array}{ccccc}
A_{1}^{(0)} & A_{0}^{(0)} & & & \\
A_{2}^{(1)} & A_{1}^{(1)} & A_{0}^{(1)} & & \\
& \ddots & \ddots & \ddots & \\
& & A_{2}^{(K-1)} & A_{1}^{(K-1)} & A_{0}^{(K-1)} \\
& & & A_{2}^{(K)} & A_{1}^{(K)}
\end{array}\right]
$$




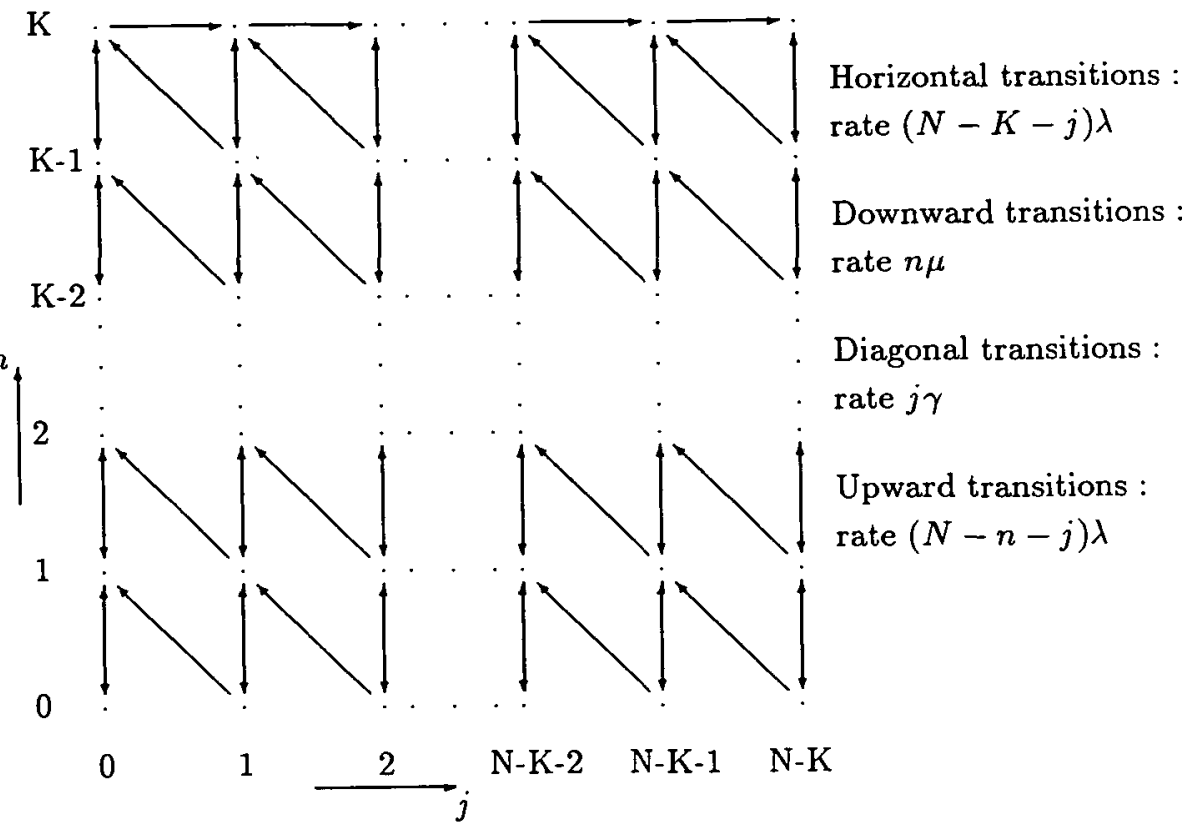

Figure 2. State transition diagram of the restricted system.

where

$$
\begin{aligned}
& A_{0}^{(n)}=\left[\begin{array}{cccc}
(N-n) \lambda & & & \\
\gamma & (N-n-1) \lambda & & \\
\ddots & \ddots & & \\
& (N-K-1) \gamma & (K-n+1) \lambda & \\
& & (N-K) \gamma & (K-n) \lambda
\end{array}\right] \quad 1 \leqslant n \leqslant K-1 \\
& A_{\mathrm{i}}^{(K)}=\left[\begin{array}{cccc}
-((N-K) \lambda+K \mu) & (N-K) \lambda & & \\
& -((N-K-1) \lambda+K \mu) & (N-K-1) \lambda & \\
\ddots & -(\lambda+K \mu) & \lambda \\
& & & -K \mu
\end{array}\right]
\end{aligned}
$$

$A_{2}^{(n)}=n \mu I, 1 \leqslant n \leqslant K, I$ the identity matrix and $A_{1}^{(n)}$ is a diagonal matrix with elements $\left[A_{1}^{(n)}\right]_{j}=-((N-n-j) \lambda+n \mu+j \gamma)$ for $0 \leqslant n \leqslant K-1,0 \leqslant j$ $\leqslant N-K$, with all matrices of order $(N-K+1) \times(N-K+1)$.

So the balance equations may be written in the form

$$
\begin{array}{r}
\mathbf{p}_{0} A_{1}^{(0)}+\mathbf{p}_{1} A_{2}^{(1)}=0 \\
\mathbf{p}_{n-1} A_{0}^{(n-1)}+\mathbf{p}_{n} A_{1}^{(n)}+\mathbf{p}_{n+1} A_{2}^{(n+1)}=0 \\
\mathbf{p}_{K-1} A_{0}^{(K-1)}+\mathbf{p}_{K} A_{1}^{(K)}=0
\end{array}
$$

where $\mathbf{p}_{n}=\left(p_{n 0}, p_{n 1}, \ldots, p_{n, N-K}\right)$. 
The solution of this system is given by (see Gaver et al. [1])

$$
\begin{aligned}
\mathbf{p}_{K} C_{K} & =0 \\
\mathbf{p}_{n} & =\mathbf{p}_{n+1} A_{2}^{(n+1)}\left(-C_{n}^{-1}\right), \quad 0 \leqslant n \leqslant K-1 \\
\sum_{n=0}^{K} \mathbf{p}_{n} \mathbf{e} & =1
\end{aligned}
$$

where

$$
\begin{aligned}
& C_{0}=A_{1}^{(0)} \\
& C_{n}=A_{1}^{(n)}+A_{2}^{(n)}\left(-C_{n-1}^{-1}\right) A_{0}^{(n-1)} \quad 1 \leqslant n \leqslant K
\end{aligned}
$$

and $\mathbf{e}^{t}=(1,1, \ldots, 1)$. Gaver, et al $[1]$, show that the matrices $C_{n}$ are all invertible.

When the process is in state $(n, j)$ the total rate at which attempts to weld are made is $(N-n-j) \lambda+j \gamma$, whereas the rate at which first attempts to weld are made is $(N-n-j) \lambda$. Rejected attempts occur only when $n=K$ and are made at rate $(N-K-j) \lambda$.

Thus, the probability of an unsuccessful attempt to weld is given by

$$
\frac{\sum_{j=0}^{N-K} p_{K_{J}}[(N-K-j) \lambda+j \gamma]}{\sum_{i=0}^{K} \sum_{j=0}^{N-K} p_{i j}[(N-i-j) \lambda+j \gamma]} .
$$

The numerator of (3.5) is the expected rate at which rejected attempts occur, while the denominator is the expected rate at which all attempts are made.

It is also of interest to calculate the probability of an unsuccessful first attempt, that is an attempt by a welder not currently trying again after an unsuccessful attempt. This is given by

$$
\frac{\sum_{j=0}^{N-K} p_{K j}(N-K-j) \lambda}{\sum_{i=0}^{K} \sum_{j=0}^{N-K} p_{i j}(N-i-j) \lambda} .
$$

The numerator of (3.6) is the expected rate at which first attempts are rejected, while the denominator is the expected rate at which first attempts are made.

\section{Numerical results}

A welding shop would typically have between 10 and 20 operators. The maximum number of effective simultaneous welds depends on the design of the power supply, but could be in the range 3 to 5 . The duration of a spot weld is about 0.4 seconds, while a given operator may perform welds at 5 to 20 spots per 100 seconds. We shall assume the reattempt rate is 1 try per second. Tables $1,2,3$ and 4 give, for different $N$ and $K$, the values of (a) the probability of defective 
welds in the unrestricted system $\left(N_{b} / N_{w}\right)$, (b) the rejection probability in the restricted system (3.5) and (c) the first attempt rejection probability in the restricted system (3.6). In all the tables, $\mu=2.5$ and $\gamma=1$ while $\lambda$ ranges from 0.05 to 0.2 .

\begin{tabular}{|r|c|c|c|c|}
\hline & $\mathrm{K}=1$ & $\mathrm{~K}=2$ & $\mathrm{~K}=3$ & $\mathrm{~K}=5$ \\
\hline $\mathrm{N}=10$ (b) & 0.163244736 & 0.012628787 & 0.000579510 & 0.000000342 \\
$(\mathrm{c})$ & 0.126054855 & 0.015065381 & 0.000644399 & 0.000000364 \\
\hline (a) & 0.242124979 & 0.029919969 & 0.002333318 & 0.000005004 \\
$\mathrm{~N}=15$ (b) & 0.335720521 & 0.035817501 & 0.002570483 & 0.000005264 \\
$(\mathrm{c})$ & 0.268137348 & 0.029230348 & 0.002234599 & 0.000004854 \\
\hline (a) & 0.313569244 & 0.052725553 & 0.005773688 & 0.000026779 \\
$\mathrm{~N}=20$ (b) & 0.434872847 & 0.063491704 & 0.006317488 & 0.000027819 \\
$(\mathrm{c})$ & 0.359499307 & 0.051723400 & 0.005459238 & 0.000025596 \\
\hline
\end{tabular}

TABlE 1. $\lambda=0.05, \mu=2.5, \gamma=1$

\begin{tabular}{|r|c|c|c|c|}
\hline & $\mathrm{K}=1$ & $\mathrm{~K}=2$ & $\mathrm{~K}=3$ & $\mathrm{~K}=5$ \\
\hline $\mathrm{N}=10(\mathrm{a})$ & 0.297413268 & 0.044482041 & 0.004013044 & 0.000009311 \\
$(\mathrm{c})$ & 0.397180166 & 0.052771718 & 0.004391568 & 0.000009784 \\
\hline$(\mathrm{a})$ & 0.42252524922 & 0.099138873 & 0.015058499 & 0.000125825 \\
$\mathrm{~N}=15(\mathrm{~b})$ & 0.554486978 & 0.118473195 & 0.016286305 & 0.000128975 \\
$(\mathrm{c})$ & 0.483932975 & 0.097218532 & 0.013992727 & 0.000118395 \\
\hline$(\mathrm{a})$ & 0.525357581 & 0.164629337 & 0.034767168 & 0.000622074 \\
$\mathrm{~N}=20(\mathrm{~b})$ & 0.673120272 & 0.198594255 & 0.037453611 & 0.000623074 \\
$(\mathrm{c})$ & 0.615641213 & 0.164862360 & 0.031948774 & 0.000569026 \\
\hline
\end{tabular}

TABLE 2. $\lambda=0.10, \mu=2.5, \gamma=1$

\begin{tabular}{|r|c|c|c|c|}
\hline & $\mathrm{K}=1$ & $\mathrm{~K}=2$ & $\mathrm{~K}=3$ & $\mathrm{~K}=5$ \\
\hline $\mathrm{N}=10$ (b) & 0.408101548 & 0.088476372 & 0.011766326 & 0.000060373 \\
$(\mathrm{c})$ & 0.44694919 & 0.103775292 & 0.012693201 & 0.000062581 \\
\hline$(\mathrm{a})$ & 0.557699050 & 0.186166236 & 0.041268434 & 0.000755006 \\
$\mathrm{~N}=15$ (b) & 0.675105825 & 0.218814471 & 0.043995331 & 0.000754993 \\
$(\mathrm{c})$ & 0.621542623 & 0.183263588 & 0.037583034 & 0.000689636 \\
\hline (a) & 0.669487004 & 0.292702173 & 0.089238356 & 0.003458007 \\
$\mathrm{~N}=20$ (b) & 0.773490353 & 0.343758836 & 0.095062811 & 0.003352873 \\
$(\mathrm{c})$ & 0.738560682 & 0.296137255 & 0.081025682 & 0.003037951 \\
\hline
\end{tabular}

TABLE 3. $\lambda=0.15, \mu=2.5, \gamma=1$ 


\begin{tabular}{|r|c|c|c|c|}
\hline & $\mathrm{K}=1$ & $\mathrm{~K}=2$ & $\mathrm{~K}=3$ & $\mathrm{~K}=5$ \\
\hline (a) & 0.499751038 & 0.139571780 & 0.024314415 & 0.000217942 \\
$\mathrm{~N}=10$ (b) & 0.594886713 & 0.160519857 & 0.025856420 & 0.000222851 \\
(c) & 0.532888678 & 0.133527366 & 0.022220222 & 0.000204609 \\
\hline (a) & 0.659538964 & 0.278222598 & 0.079938084 & 0.002527809 \\
$\mathrm{~N}=15$ (b) & 0.737740252 & 0.315999554 & 0.083832506 & 0.002469118 \\
(c) & 0.606746600 & 0.271710745 & 0.071655024 & $0.000222440 \hat{8} 8$ \\
\hline (a) & 0.768287941 & 0.416085606 & 0.162499921 & 0.010754244 \\
$\mathrm{~N}=20$ (b) & 0.816135853 & 0.463069299 & 0.169804321 & 0.010135126 \\
(c) & 0.790300456 & 0.414048496 & 0.146166515 & 0.009116947 \\
\hline
\end{tabular}

TABLE 4. $\lambda=0.20, \mu=2.5, \gamma=1$

As expected, the probabilities of bad welds in the unrestricted case or rejection in the restricted case increase with increasing $N$ and $\lambda$, and decreasing $K$. For a welding shop to operate efficiently, these parameters must be such that either the probability of bad welds (unrestricted case) or the probability of rejection (restricted case) is small. When this happens, it may be seen from Tables 1, 2, 3 and 4 that in the restricted case the probability of rejection is of the same order as the probability of bad welds in the unrestricted case.

\section{Conclusion}

Tables $1,2,3$, and 4 indicate that it is clearly possible to equip a welding shop with a power supply the capacity of which is much smaller than the number of operators in the shop. The probabilities of interference either in the sense of causing bad welds or occasional rejections are very small for all shops where $K>2$ and are even small for $K=2$ for some parameter values.

Given the decision to use a small power supply there still remains to choose between restricting the number of simultaneous welds that are allowed as in Section 3, or allowing any number of simultaneous welds but suffering a possible loss of weld quality on a small number of welds, as in Section 2. A look at Tables $1,2,3$, and 4 shows that for comparable parameter values the probability of rejection in the restricted system is virtually the same as the probability of bad welds in the unrestricted system. However, it seems reasonable from a reliability point of view to accept an occasional rejection in order to prevent any bad welds occuring through voltage loss. Hence the restricted system would appear preferable. 


\section{Acknowledgement}

The authors would like to thank the referees for helpful comments, Tom Melville of G. M. H. Australia for posing the problem considered in this paper and Bill Henderson for valuable discussions.

\section{References}

[1] D. P. Gaver, P. A. Jacobs and G. Latouche, "Finite birth-and-death models in randomly changing environments", Adv. in Appl. Probab. 16 (1984) 715-731.

[2] D. König, "Verallgemeinerungen der Engsetschen Formel”, Math. Nachr. 28 (1964) 145-155.

[3] M. F. Neuts, Matrix geometric solutions in stochastic models: An algorithmic approach (The Johns Hopkins University Press, Baltimore, 1981). 